\title{
MOBP Regimen
}

National Cancer Institute

\section{Source}

National Cancer Institute. MOBP Regimen. NCI Thesaurus. Code C9529.

A chemotherapy regimen consisting of mitomycin, vincristine, bleomycin, and cisplatin that may be used in the treatment of cervical cancer. 